\title{
Skin Necrosis
}

National Cancer Institute

\section{Source}

National Cancer Institute. Skin Necrosis. NCI Thesaurus. Code C112114.

Death of one or more layers of skin. 